\title{
EL CONSUMO SOCIALMENTE RESPONSABLE: Un enfoque conceptual
}

\author{
Eva María MURGADO ARMENTEROS \\ Universidad de Jaén \\ emurgado@ujaen.es
}

\section{RESPONSIBLE SOCIAL CONSUMPTION: A conceptual approach}

Resumen: En este trabajo se analiza el concepto de consumo responsable, su evolución y la percepción que los consumidores españoles tienen del mismo. También se explican las diferentes formas de consumo responsable que pueden practicarse y se ofrecen algunos datos de interés sobre los hábitos del consumo responsable en España. Finalmente, se estudia la influencia que las acciones de Responsabilidad Social Corporativa pueden tener en el comportamiento de compra del consumidor.

Abstract: In this paper the concept of responsible consumption, its evolution and the perception of the spanish consumers regarding it, has been studied. Besides, it explains several ways of responsible consumption that may be practised and offers some data of interest referred to the responsible consumption habits in Spain. Finally, the influence which the corporative social responsability actions has on the shopping behaviour of the consumer is studied.

Responsabilidad Social Corporativa. Consumo Responsable. Consumidor Verde.

Palabras clave: Consumerismo. Marketing Social

Corporate Social Responsability. Responsible Consumption. Green Consumer. Consumerism. Social Marketing 


\section{Introducción}

Los países desarrollados consumen entre el 50 y 90 por 100 de los recursos naturales y generan las dos terceras partes de las emisiones de dióxido de carbono. Si se evalúa lo que un ciudadano de estos países consume en un día, en recursos como agua, energía, alimentos, ropa, etc., el resultado es una cantidad ingente con consecuencias importantes para el entorno en el que habitamos.

Sin embargo, en esta sociedad de consumo se están produciendo cambios importantes en el comportamiento de compra de los consumidores. De acuerdo con Lambin (2003), el nuevo consumidor se caracteriza por los siguientes aspectos:

- Una sensación de poder, a través de movimientos consumeristas organizados, y con gran influencia en los mercados de consumo.

- Un comportamiento de compra profesional, donde los consumidores cada vez se preocupan por estar informados para tomar decisiones más racionales e independientes de las marcas, la publicidad o las recomendaciones del vendedor. Su objetivo es obtener una buena relación calidad-precio y han pasado a ser consumidores activos.

- La relación satisfacción-placer-fidelidad. Los nuevos consumidores responsabilizan a las empresas si no quedan satisfechos o si sus quejas no reciben una respuesta adecuada, de forma que su insatisfacción no sólo se traduce en la no compra del producto, sino también en la comunicación de dicha insatisfacción a su entorno.

- La aparición de nuevas expectativas en sus relaciones con las empresas. Los consumidores desean que se les escuche, comprenda y respete. Asimismo, exigen transparencia en los precios, atributos y características de los productos.

Además, en los últimos años se ha producido un aumento de la concienciación ecológica y social de los ciudadanos. Este cambio en el sistema de valores ha sido especialmente intenso en los países desarrollados. Diversos estudios de opinión realizados en EE.UU. y Europa reflejan la existencia de una elevada sensibilidad entre los ciudadanos ante temas relacionados con la Responsabilidad Social Corporativa (RSC). En efecto, un estudio de Market \& Opinion Research International (MORI, 2004), señala que un 38 por 100 de los consumidores europeos considera que el compromiso social de una empresa es muy importante a la hora de decidir la compra de productos y/o servicios, y un 46 por 100 lo consideran bastante importante.

Esta situación está modificando las pautas de consumo en los mercados. Así, los consumidores cada vez tienen en cuenta más consideraciones de tipo social, medioambiental o ético en los procesos de compra, adoptando un enfoque de "consumo responsable".

Por su parte, las empresas deben actuar como "ciudadanos corporativos" desarrollando procesos que les permitan no sólo atender sus obligaciones sociales, medioambientes, éticas y económicas, sino impulsar, de forma voluntaria y proactiva, acciones de mejora social dirigidas a los consumidores y a la sociedad en general.

En este trabajo se profundizará en el concepto de consumo responsable, analizando su evolución, así como la percepción que los consumidores españoles tienen de dicho concepto. A continuación, se explicarán las diferentes formas de consumo responsable que puede practicar un consumidor, se analizarán algunos datos de interés sobre los hábitos del consumo responsable en España y se explicará la corriente consumerista. Finalmente, se estudiará la influencia que pueden tener las acciones de RSC llevadas a cabo por las empresas en el comportamiento de compra del consumidor. 


\section{Consumo responsable: Concepto}

No existe una definición consensuada sobre el significado de consumo responsable, utilizándose diferentes términos que hacen referencia al carácter ético, medioambiental y social que debe estar presente en el comportamiento de compra y consumo.

Siguiendo a Dueñas et al., 2014, los orígenes del consumo responsable se remontan a los años sesenta en los que se producen los primeros movimientos medioambientales y surge el denominado marketing verde. En sus inicios, el "consumo verde" se define como aquel comportamiento que trata de evitar la compra de productos: 1) que ponen en riesgo la salud del consumidor o de otras personas, 2) que suponen un daño significativo al medioambiente durante el proceso de producción y uso, 3) que consumen una cantidad excesiva de energía, 4) que usan materiales derivados de especial o ambientes amenazados, 5) que implican un maltrato innecesario de animales y afectan de manera adversa a otros países (Elkington y Hailes, 1989). En suma, el consumo verde hace referencia, fundamentalmente, a aquellos consumidores que tienen en cuenta sus preocupaciones medioambientes en los procesos de compra.

Posteriormente, el aspecto ético y moral comienza a estar presente en la toma de decisiones surgiendo los denominados "consumidores éticos" (Mintel Research, 1994).

Ambos tipos de consumo han dado paso al denominado "consumo responsable" en el que se incluyen todos los aspectos de naturaleza social que pueden influenciar el proceso de compra. Así, el consumidor no sólo tiene en cuenta aspectos medioambientales y éticos, sino también aspectos específicos como la RSC de las empresas.

En los años 70, Webster (1975, p. 188) fundamenta el concepto de consumo responsable en la idea del cuestionamiento que se plantea el consumidor con su acto de compra. En este sentido, lo define como un consumo donde "el consumidor tiene en cuenta las consecuencias públicas de su consumo privado e intenta usar su poder de compra para lograr el cambio social".

Roberts (1993, p. 140) aúna la dimensión ambiental y social definiendo al consumidor responsable como aquel "que compra productos y servicios que se percibe tienen un efecto positivo (o menos negativo) sobre el ambiente, o que favorece a las empresas que intentan generar un cambio social positivo".

La definición ofrecida por Mohr et al. (2001, p. 47), incide en la dimensión moral y la preocupación por el cambio social. En este sentido, consideran que el consumo responsable es el realizado por "una persona que basa su adquisición, uso y disposición de los productos en el deseo de minimizar o eliminar cualquier efecto dañino, y maximizar los beneficios positivos a largo plazo sobre la sociedad". Además, apuntan que la relación del consumidor con las empresas, en el acto de compra, estará influenciada por las iniciativas que dichas empresas promuevan para evitar las externalidades negativas de sus productos, así como su esfuerzo para maximizar los beneficios sociales en el corto, medio y largo plazo.

En la actualidad, el consumo responsable no sólo tiene en cuenta las dimensiones señaladas, sino también, el contexto económico y social del consumidor. En este sentido, Hailes (2007) y Akehurst et al., (2012) definen al consumidor responsable como aquella persona que, en sus actos de consumo, tiene en cuenta la oportunidad de preservar el medio ambiente y la calidad de vida en sociedad, en un contexto particular y local.

En suma, Dueñas et al. (2014) definen al consumidor socialmente responsable como aquel que rechaza los productos que suponen un riesgo para la salud, los productos con un packaging no ecológico o que desperdician energía, los productos que utilizan materiales peligrosos o provienen de hábitats o especies en peligro, que evalúa a las empresas en su comportamiento responsable y en el comercio justo, todo ello considerando el contexto particular en el cual toma sus decisiones de compra. Una síntesis del concepto de consumo responsable se refleja en la figura 1. 


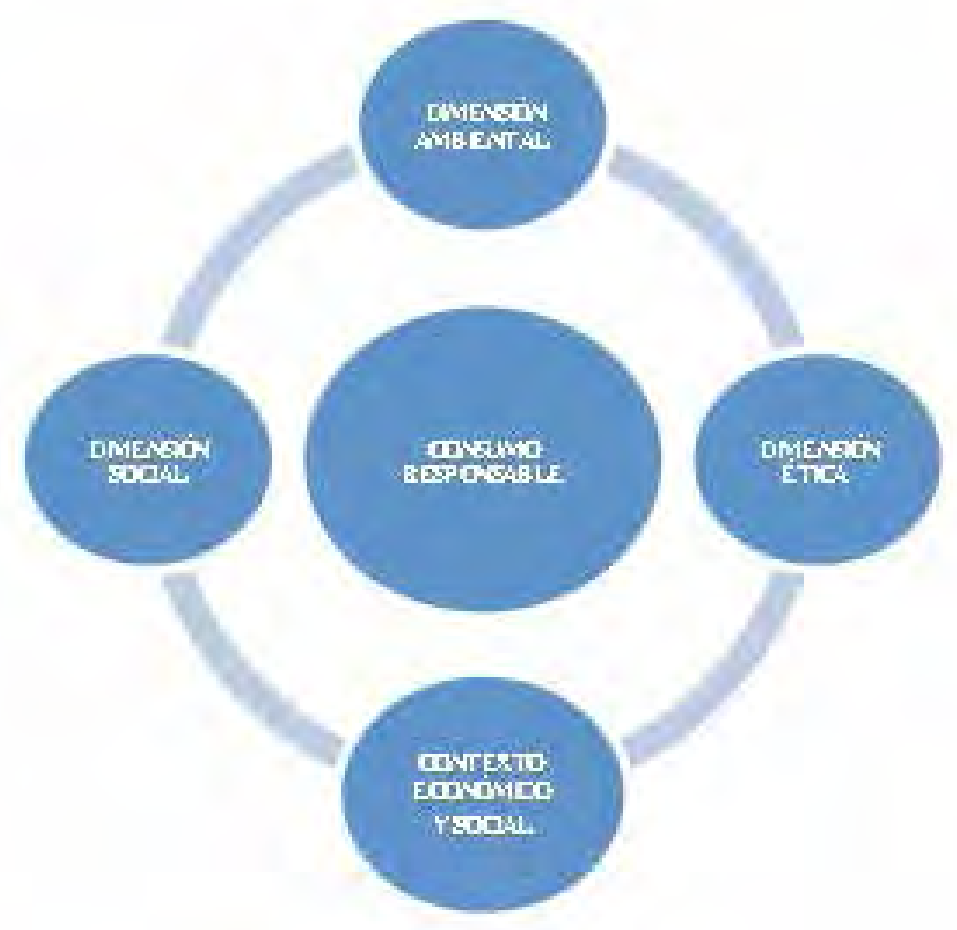

Figura 1. Concepto de consumo responsable (Fuente: Elaboración propia).

El estudio sobre consumo responsable y desarrollo sostenible (2012), realizado por el Club de Excelencia en Sostenibilidad, recoge la opinión de los consumidores españoles sobre su percepción acerca de lo que significa el consumo responsable. En este sentido, se asocia a la idea de ahorro, austeridad, control del gasto. En definitiva, los consumidores españoles consideran que se trata de "consumir aquello que verdaderamente se necesita, pero sin derrochar".

Asimismo, se relaciona con el compromiso y los valores sociales presentes en la sociedad. En este sentido, se destaca la preocupación por cuestiones relacionadas con el medio ambiente, la ecología, el control del gasto en agua y energía, y las condiciones laborales de las personas que intervienen en la producción de los bienes y servicios.

Una síntesis del significado de consumo responsable para el consumidor español se recoge en la tabla 1, donde se presentan las principales manifestaciones realizadas por los consumidores en relación con lo que significa este concepto.

\section{Comportamiento del consumidor responsable}

\subsection{Formas de consumo responsable}

Partiendo del concepto de consumo socialmente responsable, las principales razones que llevan a un consumidor a actuar de este modo se pueden concretar en las siguientes:

- Contribuir activamente a hacer efectivos los derechos de información, elección y reclamación de los consumidores.

- Solidaridad y respeto de todas las personas implicadas en el proceso productivo.

- Proteger el medio ambiente. 
- Ejercer un consumo responsable utilizando el poder de influencia que tiene el consumidor para cambiar la realidad económica.

- Contribuir a la generación de un nuevo mundo, sostenible y responsable.

\begin{tabular}{|l|l|l|}
\hline Componentes & Descripción & Importancia \\
\hline $\begin{array}{l}\text { PAUTAS Y HÁBITOS QUE } \\
\text { RIGEN LO COTIDIANO }\end{array}$ & $\begin{array}{l}\text {-Comprar lo que necesita. } \\
\text {-No exceso, ni derroche. } \\
\text {-Ser consciente del efecto medioambiental. } \\
\text {-Freno al consumo. } \\
\text {-Otras formas de compra y consumo. }\end{array}$ & \\
\hline $\begin{array}{l}\text { HOGAR, ECONOMÍA } \\
\text { DOMÉSTICA }\end{array}$ & $\begin{array}{l}\text {-Agua, electricidad, gas, calefacción (coste } \\
\text { energía, consumo). } \\
\text {-Valores: "se lo digo a mis hijos". } \\
\text {-Administración, enfermedad, } \\
\text { electrodomésticos, lámpara de bajo } \\
\text { consumo, etc. }\end{array}$ & \\
\hline ENERGÍA Y AGUA & -Ahorro. & $19 \%$ \\
\hline OTROS & $\begin{array}{l}\text {-Transporte: carburante, público/privado, } \\
\text { conducción eficiente. } \\
\text {-Actividad laboral/empresarial. } \\
\text {-Educación, formación. } \\
\text {-Ideológica: capitalismo, crisis. }\end{array}$ & $16 \%$ \\
\hline
\end{tabular}

Tabla 1. Percepción del significado de consumo responsable para el consumidor español. Respuestas múltiples (Fuente: Club de Excelencia en Sostenibilidad, 2012).

En línea con estas razones, y tomando como base la Guía para un Consumo Responsable elaborada por Ausborn (2006), las prácticas que un consumidor responsable puede desarrollar se concretan en las siguientes:

- Compra de productos y servicios sostenibles.

- Evitar residuos y aplicar la regla de las $3 \mathrm{R}$ : reducir, reutilizar y reciclar.

- Contribuir a generar una empresa social y medioambiental más responsable.

- Tener una participación más activa en prácticas y actividades de responsabilidad social.

\section{Compra de productos y servicios sostenibles.}

La adquisición de productos sanos y seguros es un derecho básico que tienen los consumidores correspondiendo a las instituciones públicas y a las empresas la responsabilidad de que se cumpla dicho derecho.

Sin embargo, el consumidor también debe preocuparse por el cumplimiento de este principio y velar no sólo porque los productos adquiridos sean saludables y seguros para sí mismo, sino también para todas aquellas personas implicadas en el proceso de fabricación del producto y para nuestro planeta, en general.

El consumo de energías renovables procedentes de la energía solar, geotérmica, eólica, hidráulica, y la obtenida a partir de biomasa residual, es otra de las acciones que se plantean con la finalidad de eliminar la energía nuclear y la basada en la quema de combustibles fósiles, principales causas del cambio climático. La liberalización del sector energético permite que el consumidor pueda optar por estas energías alternativas. Además, debe realizar un uso racional y eficiente de los recursos energéticos. 
La compra de productos de comercio justo es una alternativa solidaria que permite asegurar los derechos de productores y trabajadores marginados, buscando una mayor equidad en el comercio internacional.

La inversión socialmente responsable es una forma de plantear el ahorro donde el consumidor busca tanto un rendimiento económico como una rentabilidad social. Este tipo de inversión se materializa a través de fondos de inversión, planes de pensiones éticos, o depósitos que garantizan que el dinero del consumidor se destinará a financiar proyectos empresariales sostenibles.

La adquisición de productos y/o servicios de personas con dificultad de acceso al mercado laboral (discapacitados y personas en riesgo de exclusión social) es otro de los comportamientos responsables que pueden adoptarse.

Las iniciativas de turismo responsable buscan el equilibrio entre el beneficio económico, el desarrollo social, la preservación y contacto respetuoso con el destino turístico y el cuidado del medio ambiente. La contratación de servicios turísticos a empresas responsables y la adopción de un comportamiento responsable en los destinos visitados, son otras de las acciones que pueden implementarse.

En la tabla 2 se recogen los principales beneficios que pueden derivarse de la adquisición de bienes y servicios socialmente responsables.

\begin{tabular}{|l|l|}
\hline Tipos & Beneficios de un consumo responsable \\
\hline $\begin{array}{l}\text { Adquisición de productos sanos y } \\
\text { seguros }\end{array}$ & $\begin{array}{l}\text {-Alimentos: prevención de enfermedades crónicas. La } \\
\text { compra de productos locales y de temporada contribuye a } \\
\text { ahorrar energía y fortalecer la infraestructura local. }\end{array}$ \\
-Productos ecológicos: apoyo a la agricultura más \\
sostenible. \\
-Medicamentos: utilizarlos según las indicaciones para \\
impedir que se creen resistencias no deseadas. \\
-Ropa fibras naturales: reduce la contaminación, fomenta \\
una producción más sostenible y segura.
\end{tabular}

Tabla 2. Principales beneficios de la compra de productos y servicios sostenibles (Fuente: Elaborado a partir de Ausborn, 2006). 


\section{Evitar residuos y la regla de las $3 \mathbf{R}$.}

El consumo excesivo y la cultura actual de "usar y tirar" han originado un nivel insostenible de residuos que se generan a lo largo del proceso productivo: desde la fase de aprovisionamiento de materias primas, producción, comercialización y la posterior eliminación o recuperación de los residuos.

Para hacer frente a este problema se ha establecido la regla de las 3 R: Reducir (ahorrando materias primas), Reutilizar (alargando la vida útil de los materiales) y Reciclar (o reintroducir en el sistema productivo).

A través de esta práctica se contribuiría a la protección del medio ambiente y se frenaría el agotamiento de los recursos naturales.

\section{Contribuir a generar una empresa social y medioambiental más responsable.}

El reconocimiento, por parte de los consumidores, de las prácticas de RSC llevadas a cabo por las empresas, es otra de las formas de contribuir al consumo responsable. Para ello, adquiere especial relevancia la transparencia empresarial, esto es, que la empresa proporcione, verifique y comunique al consumidor información actualizada de todos los procesos de la cadena de valor y de sus prácticas de RSC. Esta información puede permitir al consumidor seleccionar un producto y/o servicio atendiendo a criterios de impacto en la salud, el medio ambiente y la sociedad.

En línea con esta práctica, el estudio de opinión y valoración de los consumidores sobre la Responsabilidad Social de la Empresa en España (2010), revela que el 56,9 por 100 de los consumidores manifiestan no tener en cuenta información sobre el comportamiento social y medioambiental de la empresa cuando van a comprar un producto y/o servicio. A pesar de que la proporción de consumidores que no consultan este tipo de información es mayoritaria, se observa un ligero incremento del porcentaje de consumidores que siempre tienen en cuenta esa información, situándose en el 8,3 por 100 .

También se ha incrementado el porcentaje de consumidores que estaría dispuesto a optar en sus compras por las empresas con un mejor comportamiento social y medioambiental, situándose en el 59, 5 por 100. Sin embargo, se reduce el porcentaje de consumidores que está dispuesto a asumir mayores costes (10,5 por 100), lo que se justifica no por un cambio de actitud, sino por la situación económica actual.

Centrándonos en la compra de bienes producidos y comercializados de forma responsable, el 10,5 por 100 de los entrevistados manifiestan no comprar este tipo de bienes, frente a un 46,7 por 100 de los consumidores para los que este tipo de productos suponen entre un 5 y un 15 por 100 de sus adquisiciones.

Por otra parte, el 70,5 por 100 de los consumidores españoles declaran no haber realizado ninguna acción que exprese su desacuerdo o protesta con respecto a la conducta inadecuada de las empresas. Entre los consumidores más activos, las acciones de consumo responsable más frecuentes que se han destacado son: el reenvío de información a amigos/ familiares sobre el comportamiento de la empresa, la firma de algún tipo de manifiesto relacionado con la mala conducta de alguna empresa, el envío de peticiones a alguna empresa para que cambie alguna práctica y el envío de peticiones a los poderes públicos para que ejerzan mayor control sobre las empresas.

\section{Participación más activa en actividades de responsabilidad social.}

Aparte de adquirir productos y servicios socialmente responsables, el consumidor puede participar activamente en actividades de responsabilidad social que van más allá del consumo. Entre estas actividades cabe destacar las prácticas de voluntariado (colaborando con organizaciones no gubernamentales, asociaciones y fundaciones en proyectos y actividades 
que apoyan causas sociales), los bancos del tiempo (en los que se intercambian servicios por servicios que no se pagan con dinero, sino en unidad de tiempo) y las redes de intercambio (basadas en el intercambio de bienes, conocimientos y servicios sin intermediación del dinero.

A través de estas actividades se contribuirá a crear un mundo más solidario, fomentando las redes sociales entre los ciudadanos.

\subsection{Tipología de consumidores responsables}

Las actitudes y motivaciones que tienen los consumidores españoles hacia el consumo responsable permiten distinguir tres tipologías (Estudio de Consumo Responsable y Desarrollo Sostenible, 2012):

- Conservadores: aquellos que vinculan responsabilidad y ahorro. Reciclan, se preocupan por el medio ambiente y adquieren marca blanca en sus compras cotidianas.

- Progresistas: no sólo tienen en cuenta la responsabilidad y el ahorro asumiendo, además, los efectos que sus comportamientos suponen para el medio ambiente. Se preocupan no sólo por el reciclado y la optimización de la compra, sino también por otros temas relacionados con la RSC como las políticas de igualdad, la conciliación familiar, y su papel como ciudadanos sociales que deben exigir responsabilidad a los poderes políticos y a la sociedad, en general. - Individualistas: aúnan responsabilidad y ahorro, son conscientes de la importancia del reciclado pero adoptan un papel inactivo justificando su actuación con pretextos basados en la ineficacia de las administraciones y entes públicos.

\section{EI movimiento consumerista}

En el marco del consumo responsable, surge la corriente de "consumerismo" en los mercados de consumo para realizar boicots contra marcas y/o productos que no cumplen con los criterios de RSC.

En este contexto, el consumerismo puede definirse como un movimiento social, de la ciudadanía, que trata de aumentar y defender los derechos y el poder de los compradores respecto a los productores.

La dificultad para evaluar los productos y servicios, la frustración producida por el desfase entre las expectativas y el resultado del funcionamiento de los productos, la existencia de determinadas prácticas fraudulentas de marketing, el deterioro progresivo del medio ambiente, y la desilusión con el sistema social e institucional, son algunos de los antecedentes señalados en la literatura acerca de este movimiento. En la actualidad, parte de estos factores están siendo atendidos por las empresas, mediante la legislación o a través de la creación de departamentos de atención al cliente.

La insatisfacción del consumidor con las prácticas de mercado aplicadas por algunas empresas constituye el principal motor de activación del consumerismo. En este sentido, García (1997) señala que dicha insatisfacción puede originar cuatro alternativas de respuesta por parte del consumidor:

1. Buscar soluciones fuera del sistema tradicional de marketing: mediante auto organizaciones consumeristas basadas en el trueque y en el intercambio de productos y servicios sin utilizar el dinero como contraprestación. Las cooperativas y grupos de consumo, los bancos del tiempo y las redes de intercambio son algunas opciones. 
2. Permanecer inactivo: es una práctica en la que el consumidor adopta un papel pasivo y de conformismo con la situación.

3. Consumerismo de acción privada: el consumidor responde de forma individual resolviendo su insatisfacción en privado con actuaciones como dejar de comprar la marca o en el establecimiento en cuestión, reclamar a la empresa, comunicar su malestar a amigos y familiares.

4. Consumerismo de acción pública: el consumidor comunica su insatisfacción a una organización formal o informal de consumidores denunciándola con la finalidad de que intervengan los poderes públicos.

El consumerismo ha contribuido a mejorar las prácticas de marketing formando un grupo de presión que las empresas no pueden ignorar. Así, cada vez existe un mayor número de consumidores que no sólo reivindica sus derechos, sino que reacciona de forma organizada ante las acciones de las empresas mediante denuncias y reclamaciones, exigiendo que las relaciones de intercambio entre empresa y mercado se alejen de la soberanía del productor.

Sin embargo, las instituciones gubernamentales y la existencia de asociaciones que permitan canalizar las protestas de los consumidores, son agentes clave en el desarrollo del consumerismo. Así, las oficinas municipales de información al consumidor (OMIC), los sistemas de arbitraje y organizaciones como OCU (organización de consumidores y usuarios), entre otros, son algunos de los mecanismos que pueden utilizar los consumidores para realizar sus protestas y participar de forma activa en la defensa de sus derechos.

Algunos temas relacionados con la política de marketing de las empresas a los que ha contribuido el consumerismo son: garantía veraz, precio único, etiquetas con ingredientes, etiquetas sobre valor nutricional, productos seguros, comparación verificable, fecha de fabricación y caducidad, y publicidad veraz (Lambin, 2003).

Este movimiento ha provocado que las empresas adopten un enfoque de marketing responsable basado no en la satisfacción del consumidor a corto plazo, sino en la generación de valor y en su bienestar a largo plazo.

\section{Influencia de la responsabilidad social corporativa en el comportamiento de compra}

Las acciones que realiza la empresa en materia de responsabilidad social corporativa (RSC, en adelante) también pueden repercutir en los consumidores, modificando sus actitudes, comportamientos de compra y educando su forma de consumo. Esto explica que, desde finales de los años 80, se venga desarrollando una línea de investigación en marketing que analiza la influencia que, sobre el comportamiento del consumidor, tiene la información que recibe éste sobre las prácticas que realizan las empresas en materia de RSC. Sin embargo, los trabajos existentes no son concluyentes y mientras algunos revelan que no es relevante la influencia de la información de RSC sobre la intención de compra (Bigné et al., 2005, Bigné y Currás, 2008), otros han demostrado que existe una influencia positiva (Fernández y Merino, 2005)

En relación con los trabajos en los que se han encontrado evidencias de que la información sobre RSC puede influir en la intención de compra, parece existir consenso en que las percepciones de los consumidores sobre las prácticas de RSC de las empresas tienen una influencia positiva en: 1) la imagen corporativa, 2) las actitudes hacia la empresa, 3) la reputación corporativa, y 4) la evaluación que realizan los consumidores sobre los atributos del producto. En suma, el valor añadido que se genera a través de las actividades de RSC puede suponer una ventaja competitiva importante para aquellas empresas socialmente responsables (Du et al., 2007), de modo que los consumidores pueden tener actitudes más positivas e intenciones de compra más fuertes hacia aquellos productos y/o servicios de empresas que apliquen prácticas de RSC.

Las diferencias existentes entre los consumidores en función de la valoración que reali- 




Figura 2. Factores clave en la consideración de la RSC en el proceso de compra del consumidor. Fuente: Öberseder et al., (2011).

zan de las prácticas de RSC, ha llevado a la consideración de esta variable como un posible criterio para segmentar el mercado. No obstante, el segmento de consumidores que tiene en cuenta la RSC en su comportamiento de compra, todavía es pequeño (Mohr et al., 2001). Por tanto, aunque el compromiso de la empresa con la RSC es valorado cada vez más por los consumidores, no es un criterio dominante en las decisiones de compra (Öberseder et al., 2011). En este sentido, los consumidores sitúan la RSC como la dimensión menos importante en la decisión de compra, prevaleciendo otros criterios como el precio, la calidad, o la marca (Beckmann et al., 2005).

Al margen de esta conclusión, diversos estudios señalan que las mujeres parecen tener una mayor preocupación por los temas de RSC que los hombres, y que la edad, el nivel de estudios y la capacidad adquisitiva también sugieren diferencias significativas en la valoración que se realiza de las actividades de RSC. En este sentido, a mayor edad, nivel de estudios y capacidad adquisitiva, existe mayor preocupación por los temas de responsabilidad social (Beckmann et al., 2005; Wagner et al., 2008).

En línea con Bhattacharya y Sen (2004), parece existir una relación positiva entre RSC y comportamiento de compra cuando se cumplen una serie de condicionantes: 1) el consumidor apoya los esfuerzos de RSC de la empresa, 2) se produce una alta adecuación de la causa con la empresa, 3) el producto es de alta calidad, y 4) no se le pide al consumidor que pague una prima o mayor precio por la responsabilidad social.

La influencia de las actividades de RSC en el proceso de compra del consumidor dependerá de diversos factores. En este sentido, Öberseder et al., (2011) distinguen 3 factores clave (figura 2) ordenados jerárquicamente: 1) la información y preocupación individual que tenga el consumidor sobre los temas de RSC, 2) la situación económica/financiera del individuo y 3) la imagen de la empresa, la credibilidad de las iniciativas en materia de RSC y la influencia de los grupos de pertenencia. Por tanto, un consumidor no valorará las actividades de RSC, sino recibe información de la empresa sobre estas actividades o está preocupado por dichos temas, si su situación financiera le impide pagar el sobreprecio por la aplicación de dichas políticas, y si la imagen de la empresa o la credibilidad respecto a dichas acciones es débil o dudosa.

\section{Bibliografía}

Akehurst, G., Alfonso, C., Martins Gonçalves, H. (2012). Re-examining green pur-chase behaviour and the green consumer profile: New evidences. Management Decision, 50 (5): 972-988. 
Ausborn, B. (2006). Guía para un consumo responsable. El papel de los y las consumidoras en la Responsabilidad Social de la Empresa. Observatorio de la Responsabilidad Social Corporativa, Madrid.

Bhattacharya, C.B., Sen, S. (2004). Doing better at doing good: when, why, and how consumers respond to corporate social iniciatives. California Management Review, 47, (1): 9-24.

Bigné, E., Chumpitaz R., Andreu, L., Swaen, V. (2005). Percepción de la responsabilidad social corporativa: un análisis cross-cultural. Universia Business Review, Primer Trimestre: 14-27.

Bigné, E. ,Currás, R. (2008). Influye la imagen de responsabilidad social en la intención de compra? El papel de la identificación del consumidor con la empresa. Universia Business Review, Tercer Trimestre: 10-23.

Beckmann, S.C., Bigné, E., Andreu, L., Chumpitaz, R., Swaen, V. (2005). An international comparison of corporate social responsibility perceptions. World Marketing Conference, Muenster, Germany.

Confederación de Consumidores y Usuarios (2010). RSE 2010. La opinión y valoración de los consumidores sobre la Responsabilidad Social de la Empresa en España. Madrid.

Club de Excelencia en Sostenibilidad (2012). Consumo Responsable y Desarrollo Sostenible. Tendencias de consumo responsable 2012. Madrid.

Du, S. Bhattacharya, C.B., Sen, S. (2010). Maximizing business returns to corporate social responsibility (CSR): The role of CSR Communication. International Journal of Management Review, 12, (1): 8-19.

Du, S. Bhattacharya, C.B., Sen, S. (2007). Reaping relational rewards from corporate social responsibility: The role of competitive positioning. International Journal of Research in Marketing, 24, (3): 224-241.

Dueñas, S. Perdomo-Ortiz, J., Villa, L. E. (2014). El concepto de consumo socialmente responsable y su medición. Una revisión de la literatura. Estudios Gerenciales: 1-14. Disponible en: http://dx.doi.org/10.1016/j.estger.2014.01.022

Elkington, J., Hailes, J. (1989). The green consumer guide: From shampoo to cham-pagne: Highstreet shopping for a better environment. London: V. Gollancz.

Fernández, D., Merino, A. (2005). ¿Existe disponibilidad a pagar por responsabilidad social corporativa? Percepción de los consumidores. Universia Business Review, Tercer Trimestre: 2-17.

García, I. (1997). Una introducción al debate Marketing-consumerismo. Facultad de Ciencias Económicas y Empresariales, Universidad de Deusto, San Sebastián.

Hailes, J. (2007). The New Green Consumer Guide. UK: Simon y Schuster Ltd.

Lambin, J. J. (2003). Marketing estratégico. ESIC, Madrid.

Mohr, L. A., Webb, D. J., Harris, K. E. (2001). Do consumers expect companies tobe socially responsible? The impact of corporate social responsibility on buyingbehavior. Journal of Consumer Affairs, 35 (1): 45-72.

Mintel Research. (1994). The Green Consumer (vol. 1 \& 2). London: Mintel Research.

Moyano, E., Navarro, C. (2004). El consumerismo como respuesta activa de los consumidores. Distribución y Consumo, Marzo-Abril: 78-80.

Öberseder, M. Schlegelmilch, B.B., Gruber, V. (2011). Why don't consumers care about CSR?: A qualitative study exploring the role of CSR in consumption decision. Journal of Business Ethics, 104, (4): 449-460.

Roberts, J. A. (1993). Sex differences in socially responsible consumers' behavior. Psychological Reports, 73 (1): 139-148.

Wagner, T. Bicen, P., Hall, Z.R. (2008). The dark side of retailing: towards a scale of corporate social irresponsibility. International Journal of Retail \& Distribution Management, 36, 2: 124142.

Webster, F. E. (1975). Determining the characteristics of the socially conscious con-sumer. Journal of Consumer Research, 2 (3): 188-196.

\section{3}

